\title{
Review
}

\section{Farnesoid $X$ receptor: a master regulator of hepatic triglyceride and glucose homeostasis}

\author{
Yang JIAO, \#, Yan LU, \#, Xiao-ying $\mathrm{LI}^{1,2, *}$ \\ ${ }^{1}$ Shanghai Institute of Endocrinology and Metabolism, Department of Endocrine and Metabolic Diseases, Shanghai Clinical Center \\ for Endocrine and Metabolic Diseases, Ruijin Hospital, Shanghai Jiao Tong University School of Medicine, Shanghai 200025, China; \\ ${ }^{2}$ The Key Laboratory of Endocrine Tumors and the Division of Endocrine and Metabolic Diseases, E-Institute of Shanghai Universities, \\ Shanghai 200025, China
}

\begin{abstract}
Non-alcoholic fatty liver disease (NAFLD) is characterized by the aberrant accumulation of triglycerides in hepatocytes in the absence of significant alcohol consumption, viral infection or other specific causes of liver disease. NAFLD has become a burgeoning health problem both worldwide and in China, but its pathogenesis remains poorly understood. Farnesoid X receptor (FXR), a member of the nuclear receptor (NR) superfamily, has been demonstrated to be the primary sensor for endogenous bile acids, and play a crucial role in hepatic triglyceride homeostasis. Deciphering the synergistic contributions of FXR to triglyceride metabolism is critical for discovering therapeutic agents in the treatment of NAFLD and hypertriglyceridemia.
\end{abstract}

Keywords: farnesoid X receptor; non-alcoholic fatty liver disease; liver; triglyceride metabolism; glucose metabolism; drug discovery

Acta Pharmacologica Sinica (2015) 36: 44-50; doi: 10.1038/aps.2014.116; published online 15 Dec 2014

\section{Introduction}

Non-alcoholic fatty liver disease (NAFLD) is characterized by the aberrant accumulation of triglycerides (TGs) in hepatocytes in the absence of significant alcohol consumption, viral infection, or other specific causes of liver disease. NAFLD currently affects $30 \%$ of Western individuals and $15 \%$ of the Chinese population ${ }^{[1,2]}$. The disease comprises a spectrum of hepatic histological abnormalities, ranging from simple steatosis to steatohepatitis and cirrhosis, and may even progress to hepatocellular carcinoma. In addition, NAFLD is strongly associated with several cardiometabolic abnormalities, including type 2 diabetes mellitus (T2DM) and coronary heart disease $\mathrm{e}^{[3,4]}$. For instance, our previous study found that NAFLD was associated with atherosclerosis in middle-aged and elderly Chinese individuals, independent of conventional cardiovascular disease risk factors or metabolic syndrome ${ }^{[5]}$.

The hallmark of NAFLD is intrahepatic TG accumulation, which results from an imbalance between the hepatic TG input and output. In the liver, TGs are primarily synthesized from two sources. (1) De novo lipogenesis (DNL), which is a complex process involving multiple metabolic reactions that begin

\footnotetext{
\#These authors contributed equally to this work.

* To whom correspondence should be addressed.

E-mail lixy@sibs.ac.cn

Received 2014-06-29 Accepted 2014-09-01
}

with acetyl-coenzyme A (CoA). The rate of this process is tightly regulated by several nuclear transcription factors, such as sterol regulatory element binding protein 1c (SREBP-1c). SREBP-1c promotes DNL via binding to the promoter regions of lipogenic enzyme genes and enhancing their transcription. (2) Fatty acid (FA) uptake. FAs derived from the lipolysis of adipose tissues are released into the circulation and then transported to the liver by the hepatic artery and portal vein. Membrane proteins such as fatty acid transporter proteins (FATPs) direct the FA traffic from the circulation to the liver. Additionally, FAs in the liver have two main fates. (1) $\beta$-Oxidation, in which FAs can be oxidized in the mitochondria to produce ketone bodies, which are exported to provide fuel for other tissues. (2) Very low-density lipoprotein (VLDL) export, in which TGs can be assembled with a newly synthesized apoprotein B-100 (apoB-100) and secreted into the systemic circulation as a constituent of VLDLs ${ }^{[6]}$. Liver steatosis occurs when the TG homeostasis is disrupted due to an increase in DNL and FA uptake and a reduction in FA oxidation and VLDL export. However, the molecular mechanisms involved in the development of NAFLD remain poorly elucidated.

\section{FXR gene and protein structure}

Farnesoid X receptor (FXR, NR1H4), a member of the nuclear receptor superfamily, was originally cloned from a rat liver cDNA library in $1995^{[7,8]}$. FXR was named on the basis of its 
weak activation by supraphysiological levels of farnesoid, an intermediate of mevalonate metabolism ${ }^{[7]}$. Subsequent studies have demonstrated that specific bile acids (BAs), such as chenodeoxycholic acid (CDCA) and cholic acid (CA), are endogenous ligands for FXR ${ }^{[9-11]}$. There are two FXR genes: FXRa and FXR $\beta$. FXR $\beta$ is a pseudogene in humans, although it serves as a lanosterol sensor and encodes a functional protein in rodents, rabbits and dogs ${ }^{[12]}$. Expressed from a single gene locus, FXRa encodes four isoforms (FXRa1, FXRa2, FXRa3, FXRa4) that result from two distinct promoters that initiate transcription from either exon 1 or exon 3 or the alternative RNA splicing of exon $5^{[13,14]}$. Although the four FXRa isoforms are expressed in a tissue-specific manner, the majority of the FXR target genes are regulated in an isoform-independent manner.

As a member of the NR superfamily, FXR shares a common architecture with other metabolic NRs: it is composed of an $\mathrm{N}$-terminal ligand-independent activation function 1 (AF1) domain, a highly conserved DNA binding domain (DBD) that is connected by a flexible hinge region to a moderately conserved ligand binding domain (LBD), and a C-terminal liganddependent activation function 2 (AF2) domain ${ }^{[15]}$. FXR can form an obligate heterodimer with retinoid $X$ receptor ( $R X R)$; therefore, it can be activated by both FXR and RXR ligands, such as BAs and 9-cis retinoic acid. In the absence of BAs, the FXR-RXR heterodimer binds to FXR response elements (FXREs) in the promoters of FXR target genes and in association with co-repressors, such as nuclear receptor co-repressor (NCoR), and silencing mediators for retinoic acid and thyroid hormone receptor (SMRT). Upon ligand binding, the corepressors are released and co-activators are recruited, resulting in gene transcription. The FXREs contain inverted repeats of 2 AGGTCA half sites separated by one nucleotide (IR1) ${ }^{[8,16]}$, although other variations have also been reported.

\section{FXR in hepatic triglyceride metabolism}

The relationship between BAs and TG metabolism was identified in the 1970s. The first evidence came from the observation that the administration of BAs, such as CDCA for the treatment of gallstones, resulted in decreased circulating TG levels ${ }^{[17-19]}$; conversely, patients treated with BA-sequestering resins were found to have increased serum TG and VLDL levels $^{[17,20,21]}$. Moreover, patients with monogenic familial hypertriglyceridemia displayed a defect in ileal BA absorption ${ }^{[17]}$, whereas individuals with decreased BA synthesis due to a CYP7A1 deficiency exhibited elevated serum TG concentrations ${ }^{[22]}$. These clinical observations suggest a direct relationship between BAs and TG metabolism. The importance of FXR in TG metabolism was further confirmed in FXR-deficient mice, which exhibited marked hepatosteatosis and hypertriglyceridemia $^{[23,24]}$. In addition, FXR heterozygous mice demonstrated hepatosteatosis and hyperlipidemia following shorttime high-fat diet (HFD) feeding ${ }^{[25]}$. The TG lowering effects of endogenous and synthetic FXR agonists have been evaluated in other rodent models as well. For instance, CA prevented hepatic TG accumulation and VLDL secretion in $\mathrm{KK}-\mathrm{A}(\mathrm{y})$ mice, a mouse model of hypertriglyceridemia ${ }^{[24]}$. Moreover, the synthetic FXR agonist GW4064 was able to prevent liver steatosis in obese mice, such as the $o b / o b$ and $d b / d b$ models $^{[26]}$.

At the molecular level, FXR controls TG metabolism by regulating hepatic de novo lipogenesis. The activation of FXR suppresses the expression of SREBP-1c $c^{[24]}$, a critical transcription factor that regulates hepatic TG synthesis by inducing key enzymes involved in lipogenesis, such as fatty acid synthase (FAS). The inhibition of SREBP-1c expression by FXR is mediated by a signaling cascade involving small heterodimer partner (SHP), which is able to blunt the action of liver $X$ receptor (LXR) to induce SREBP-1c expression by interacting with liver receptor homologue 1 (LRH1) ${ }^{[27]}$. However, some studies have shown that transgenic mice with hepatic SHP overexpression developed liver steatosis due to the indirect activation of the peroxisome proliferator-activated receptor $\gamma$ (PPAR $\gamma$ ) and SREBP-1c genes ${ }^{[28]}$. The deletion of SHP ameliorated the TG accumulation in $o b / o b$ mice $^{[29,30]}$, suggesting that the roles of SHP in hepatic TG metabolism are diverse and complicated. Whether SHP is required for the inhibitory roles of FXR on DNL remains to be determined in future studies. A recent study indicated that the activation of FXR induces hepatic carboxylesterase 1, which is essential for FXR-controlled lipid homeostasis $^{[31]}$.

In addition to the suppression of de novo lipogenesis, FXR activation modulates FFA oxidation and TG clearance. In human hepatocytes, FXR agonists were shown to induce the expression of PPARa and its target genes to promote FFA oxidation $^{[32-34]}$. FXR activation also inhibits the expression of microsomal triglyceride transfer protein (MTTP) via SHP, which in turn suppresses the hepatocyte nuclear factor $4 \mathrm{a}$ (HNF4a) transactivation of the MTTP promoter, thus decreasing hepatic VLDL production ${ }^{[35,36]}$. Moreover, FXR increases the activity of lipoprotein lipase (LPL) and promotes triglyceride hydrolysis by inducing APO CII expression ${ }^{[37]}$. Taken together, FXR activation reduces liver steatosis and hyperlipidemia by suppressing de novo lipogenesis and promoting TG oxidation and clearance (Figure 1A).

Given that obesity is closely associated with NAFLD, we speculate that FXR may be downregulated or dysfunctional in the livers of obese individuals. Indeed, we and others found that hepatic FXR expression is markedly reduced in obese animals and humans ${ }^{[25]}$. Mechanistic studies have further demonstrated that FXR is downregulated by the Yin Yang 1 (YY1) protein, which interrupts FXR gene transcription by binding to its first intron. In addition, we found that FXR was significantly downregulated in spontaneously aged mice ${ }^{[38]}$ due to decreased HNF1a transcriptional activity by ER stress. Together, these studies suggest that FXR downregulation may play a causal role in obesity and aging-associated fatty liver (Figure 1B). Interestingly, FXR was also found to play an important role in the pathogenesis and progression of alcoholinduced hepatic TG retention. The FXR activity was functionally impaired by chronic ethanol ingestion in a murine model of alcoholic liver disease ${ }^{[39]}$. In addition, the activation of FXR by its specific agonists, WAY-362450 or INT747, rescued the 
A

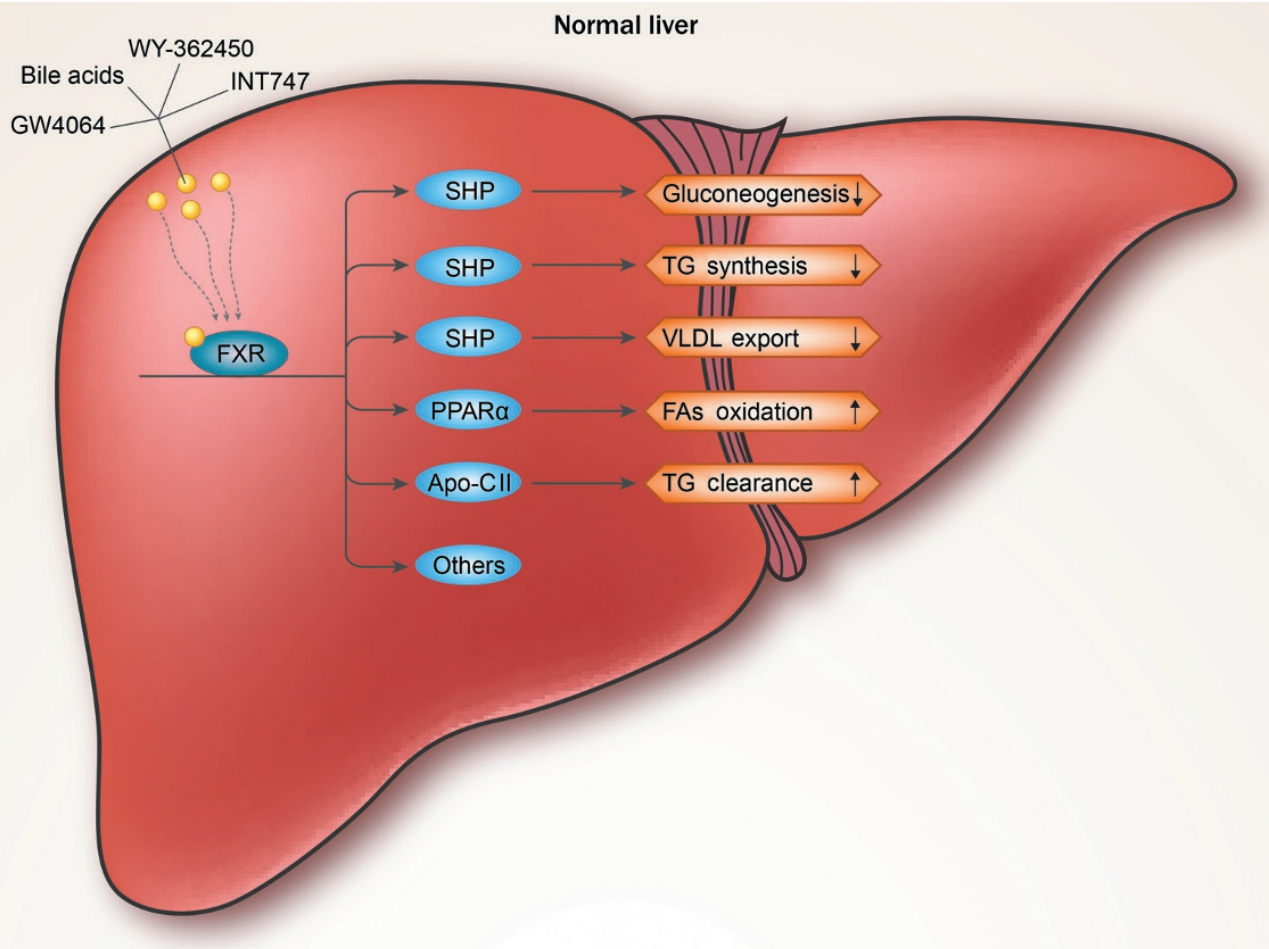

B

NAFLD

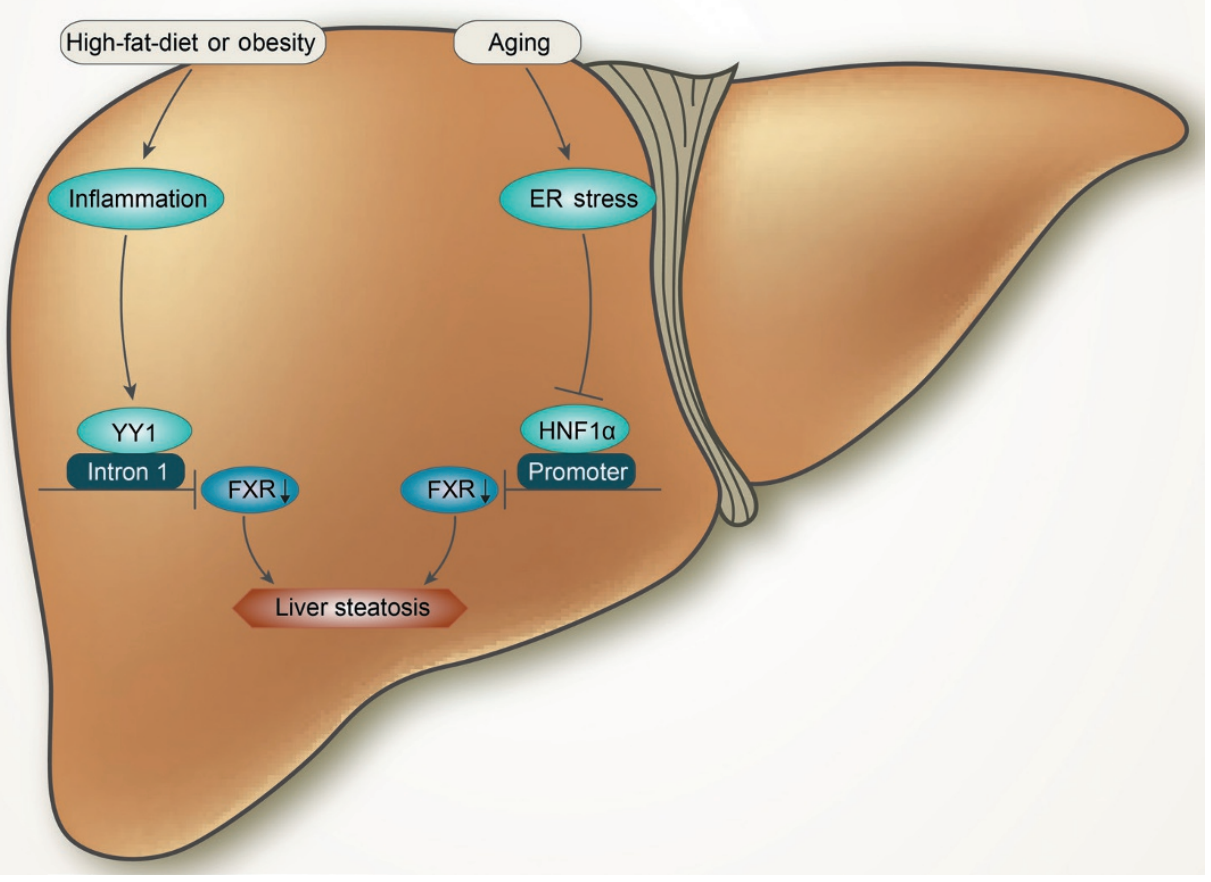

Figure 1. A schematic representation summarizing the FXR regulatory pathways in hepatic glucose and triglyceride metabolism in normal liver (A) and NAFLD (B). Bile acids or synthetic FXR agonists (GW4064, WY-362450, INT747) that activate hepatic FXR to regulate genes are important for glucose and triglyceride metabolism. FXR 1) repress gluconeogenesis, TG synthesis and VLDL export via SHP; 2) enhances the $\beta$-oxidation of FFAs via PPAR $\alpha$; and 3) promotes TG clearance via Apo-C II. The downregulation of FXR in obesity or aging results in liver steatosis. In obesity, FXR is downregulated by the Yin Yang 1 (YY1) protein, which interrupts FXR gene transcription by binding to its first intron. In the aging process, hepatic ER stress is activated, which represses FXR expression via the inhibition of the transcriptional activity of hepatocyte nuclear factor 1 alpha (HNF1 $\alpha)$.

FXR activity, thereby attenuating the ethanol-induced hepatic liver injury, steatosis and cholestasis ${ }^{[39,40]}$. In addition, the acti- vation of FXR was shown to protect against fructose-induced liver steatosis ${ }^{[11]}$, suggesting several diverse roles for FXR in 
the prevention of hepatic TG accumulation.

\section{FXR in hepatic glucose metabolism}

A physiological role for FXR in hepatic carbohydrate metabolism has emerged in recent years as well. It has been demonstrated that the activation of FXR reduces hepatic gluconeogenesis, suppresses glycolysis and induces glycogen synthesis (Figure 1A). However, a group of in vitro studies focusing on the FXR regulation of gluconeogenic enzymes showed conflicting results. Some studies showed that BAs or the non-sterol FXR agonist GW4064 inhibit PEPCK and G6Pase expression in human hepatoma cells ${ }^{[42,43]}$, while other studies demonstrated that the activation of FXR increases PEPCK expression and glucose output in primary hepatocytes ${ }^{[44,45]}$. Notably, a cholic acid-enriched diet markedly reduced the fasting blood glucose, as well as the hepatic PEPCK and G6Pase mRNA levels, in wild-type but not in FXR or SHP deficient mice, reinforcing the hypothesis that the FXR-SHP regulatory pathway represses gluconeogenesis ${ }^{[46]}$. In support of this concept, the overexpression of SHP also reduces gluconeogenic gene expression. Moreover, GW4064 treatment or the delivery of adenoviral constitutively activated FXR reduced hyperglycemia by inhibiting PEPCK and G6Pase expression in $d b / d b$ and $\mathrm{KK}-\mathrm{A}(\mathrm{y})$ diabetic mice ${ }^{[26,46]}$. At the molecular level, PEPCK and G6Pase are positively regulated by hepatocyte nuclear factor $4 a(H N F 4 a)$, glucocorticoid receptor a (GRa) and forkhead transcription factor 1 (FOXO1) in cooperation with peroxisome proliferator receptor $\gamma$ coactivator-1a (PGC1a). FXR-induced SHP expression in turn disrupts the interactions of PGC1a with the abovementioned transcription factors and subsequently leads to a decrease in gluconeogenesis ${ }^{[46]}$. In addition, FXR was shown to repress the glucose-induced transcription of several glycolytic genes, such as the liver-type pyruvate kinase gene (L-PK), by inhibiting the transcriptional activity of carbohydrate response element binding protein (ChREBP) ${ }^{[47]}$. Moreover, glycogen synthesis can also be promoted via the FXR-mediated induction of GSK3 $\beta$ phosphorylation in $d b / d b$ mice ${ }^{[26]}$. Consistent with this, the hepatic glycogen content is reduced in FXR-deficient mice ${ }^{[48]}$.

FXR also modulates insulin signaling. The activation of FXR by natural and synthetic FXR agonists or the overexpression of FXR reduces plasma glucose levels and improves insulin sensitivity in diabetic mice ${ }^{[26,46,49]}$. Conversely, the inactivation of FXR disrupts glucose homeostasis, which was observed via impaired glucose tolerance and reduced insulin sensitivity $^{[26,46,49]}$. FXR-deficient mice displayed a peripheral insulin resistance, which was reflected by a decrease in the glucose disposal rate ${ }^{[46]}$. The molecular mechanisms underlying the peripheral insulin resistance in FXR-deficient mice remain largely unknown. They may be associated with the elevated circulating FFAs and increased TG content in the peripheral tissues. Recently, several studies have suggested that the FXRmediated insulin-sensitizing effect may partially involve the induction of fibroblast growth factor 19 (FGF19) and FGF21, two newly emerged metabolic cytokines ${ }^{[50,51]}$. Given that adiponectin may mediate some of the metabolic effects of FGF21 on glucose homeostasis and insulin sensitivity ${ }^{[52]}$, further studies are still needed to investigate the relationship between FXR and adipokines, including adiponectin. Notably, a recent study showed that the effect of vertical sleeve gastrectomy (VSG) on the reduction of body weight and the improvement of glucose tolerance was significantly abolished in FXRdeficient mice, suggesting that FXR plays a crucial role in the beneficial effects of VSG ${ }^{[53]}$.

In addition, emerging studies have revealed that FXR may also regulate glucose homeostasis in other metabolic tissues. For instance, the ablation of FXR in pancreatic $\beta$ cells resulted in impaired insulin secretion. FXR-deficient mice also displayed a reduction in adipose tissue mass, lower serum leptin concentrations, and elevated plasma free fatty acid levels. Mouse embryonic fibroblasts derived from FXR-deficient mice exhibited impaired adipocyte differentiation, suggesting a direct role for FXR in adipogenesis. Consistent with this, GW4064 enhanced the insulin-stimulated glucose uptake in differentiated 3T3-L1 adipocytes ${ }^{[53]}$. In addition, FXR agonists also improved insulin resistance in the visceral preadipocytes of obese mice and rats ${ }^{[49,54]}$. FXR promotes adipocyte differentiation via the upregulation of PPARY and interrupts the Wnt/ beta-catenin pathways ${ }^{[55,56]}$. In other words, FXR also plays an important role in the regulation of adipogenesis and insulin signaling.

\section{FXR agonist development}

BAs are capable of activating FXR to different extents due to their different structural modifications. Semi-synthetic and synthetic molecules with more affinity and specificity to FXR have also been developed. GW4064 was one of the first synthetic non-steroidal FXR agonists to be developed. It has been well-established that GW4064 markedly reduces hepatic lipid accumulation and improves dyslipidemia in genetically obese mouse models, such as $o b / o b, d b / d b$ and KK-Ay mice, via SHP and SREBP-1 ${ }^{[57,58]}$. GW4064 also attenuates hepatosteatosis in high-fat-diet-induced mice by repressing the lipid transporter gene CD36 ${ }^{[59]}$. GW4064 potently improves both hyperglycemia and hyperinsulinemia, as well as increasing hepatic glycogen storage in diabetic mice ${ }^{[26]}$. GW4064 is also considered to be a modulator of multiple $\mathrm{G}$ protein-coupled receptors.

Unlike the FXR agonist GW4064, WAY-362450 offers improved oral bioavailability for mice and rats ${ }^{[60]}$. The oral administration of WAY-362450 significantly lowered the serum TG and cholesterol levels and resulted in a significant reduction in aortic arch lesions in low-density lipoprotein receptor (LDLR)-deficient mice. Additionally, the serum TG and cholesterol levels were reduced by WAY-362450 in a dose dependent manner in diabetic mice (KK-Ay and $d b / d b$ mice) and in fructose/cholesterol-fed rats ${ }^{[61]}$. WAY-362450 also significantly reduces the serum liver enzyme levels and decreases hepatic inflammation and fibrosis, as evidenced by the reduction of inflammatory cell infiltration and the hepatic gene expression of fibrosis markers ${ }^{[62]}$. However, WAY-362450 has not shown a positive impact on hepatic TG accumulation, which may limit its roles in the control of NAFLD. 
INT747 (obeticholic acid, OCA), a 6a-ethyl derivative of CDCA, is a well-known clinically developed semi-synthetic FXR agonist that has entered clinical studies for NAFLD and NASH. Recent studies have shown that INT747 is capable of modulating lipid metabolism and protects obese rodents from insulin resistance via the inhibition of hepatic lipogenesis and peripheral fatty acid synthesis ${ }^{[63-65]}$. INT747 can improve hyperglycemia and glucose tolerance by enhancing insulin signaling and suppressing hepatic gluconeogenesis ${ }^{[64,66]}$. INT747 also exerts anti-inflammatory and anti-fibrotic effects in the liver by suppressing nuclear factor (NF)-KB and subsequent hepatic satellite cell activation ${ }^{[66,67]}$.

In addition, INT747 has exhibited promising results in a phase IIa clinical trial in T2DM patients with NAFLD. The administration of 25 or $50 \mathrm{mg}$ of INT747 for 6 weeks not only improved liver enzyme and lipid accumulation but also reduced markers of liver inflammation and fibrosis. Additionally, an increase in hepatic and peripheral glucose uptake was observed during hyperinsulinemic euglycemic clamp tests.

\section{Conclusion}

FXR plays a crucial beneficial role in hepatic triglyceride homeostasis, as well as in glucose metabolism and the regulation of bile acid homeostasis. FXR can lower hepatic TG content and serum TG levels and improve insulin resistance and hyperglycemia. Therefore, FXR agonists are promising for the treatment of NAFLD, dyslipidemia and type 2 diabetes.

\section{Acknowledgements}

This study is supported by grants from the National Key Basic Research Program of China (973 Program) (№ 2012CB524902) and the National Natural Science Foundation of China (№ 81200636, 81361128009, and 81370959).

\section{Abbreviations}

NAFLD, non-alcoholic fatty liver disease; FXR, farnesoid X receptor; NR, nuclear receptor; TG, triglyceride; T2DM, type 2 diabetes mellitus; DNL, de novo lipogenesis; CoA, coenzyme A; SREBP-1c, sterol regulatory element binding protein 1c; FA, fatty acid; FATPs, fatty acid transporter proteins; VLDL, very low-density lipoprotein; apoB-100, apolipoprotein B-100; CDCA, chenodeoxycholic acid; CA, cholic acid; AF1, activation function1; DBD, DNA binding domain; LBD, ligand binding domain; AF2, activation function2; RXR, retinoid $X$ receptor; FXREs, FXR response elements; NCoR, nuclear receptor co-repressor; SMRT, silencing mediators for retinoic acid and thyroid hormone receptor; FAS, fatty acid synthase; SHP, small heterodimer partner; LXR, liver $\mathrm{X}$ receptor; LRH1, liver receptor homologue 1 ; PPAR $\gamma$, peroxisome proliferatoractivated receptor $\gamma$; PPARa, peroxisome proliferatoractivated receptor a; MTTP, microsomal triglyceride transfer protein; HNF4a, hepatocyte nuclear factor $4 a$; LPL, lipoprotein lipase; YY1, Yin Yang 1; PEPCK, Phosphoenolpyruvate carboxykinase; G6Pase, glucose-6-phosphatase; HNF4a, hepatocyte nuclear factor4a; GRa, glucocorticoid receptor $\alpha$; FOXO1, forkhead transcription factor 1; PGC1a, peroxisome proliferator receptor $\gamma$ coactivator-1 $\alpha$; L-PK, pyruvate kinase gene; ChREBP, carbohydrate response element binding protein; GSK3 $\beta$, glycogen synthase kinase $3 \beta$; FGF19, fibroblast growth factor19; VSG, vertical sleeve gastrectomy; LDLR, lowdensity lipoprotein receptor; OCA, obeticholic acid.

\section{References}

1 Szczepaniak LS, Nurenberg P, Leonard D, Browning JD, Reingold JS, Grundy S, et al. Magnetic resonance spectroscopy to measure hepatic triglyceride content: prevalence of hepatic steatosis in the general population. Am J Physiol Endocrinol Metab 2005; 288: E462-8.

2 Fan JG, Farrell GC. Epidemiology of non-alcoholic fatty liver disease in China. J Hepatol 2009; 50: 204-10.

3 Targher G, Marra F, Marchesini G. Increased risk of cardiovascular disease in non-alcoholic fatty liver disease: causal effect or epiphenomenon? Diabetologia 2008; 51: 1947-53.

4 Speliotes EK, Massaro JM, Hoffmann U, Vasan RS, Meigs JB, Sahani DV, et al. Fatty liver is associated with dyslipidemia and dysglycemia independent of visceral fat: the Framingham Heart Study. Hepatology 2010; 51: 1979-87.

5 Huang Y, Bi Y, Xu M, Ma Z, Xu Y, Wang T, et al. Nonalcoholic fatty liver disease is associated with atherosclerosis in middle-aged and elderly Chinese. Arterioscler Thromb Vasc Biol 2012; 32: 2321-6.

6 Cohen JC, Horton JD, Hobbs HH. Human fatty liver disease: old questions and new insights. Science 2011; 332: 1519-23.

7 Forman BM, Goode E, Chen J, Oro AE, Bradley DJ, Perlmann T, et al. Identification of a nuclear receptor that is activated by farnesol metabolites. Cell 1995; 81: 687-93.

8 Seol W, Choi HS, Moore DD. Isolation of proteins that interact specifically with the retinoid $X$ receptor: two novel orphan receptors. Mol Endocrinol 1995; 9: 72-85.

9 Makishima M, Okamoto AY, Repa JJ, Tu H, Learned RM, Luk A, et al. Identification of a nuclear receptor for bile acids. Science 1999; 284 : 1362-5.

10 Parks DJ, Blanchard SG, Bledsoe RK, Chandra G, Consler TG, Kliewer SA, et al. Bile acids: natural ligands for an orphan nuclear receptor. Science 1999; 284: 1365-8.

11 Wang H, Chen J, Hollister K, Sowers LC, Forman BM. Endogenous bile acids are ligands for the nuclear receptor FXR/BAR. Mol Cell 1999; 3: 543-53.

12 Otte K, Kranz H, Kober I, Thompson P, Hoefer M, Haubold B, et al. Identification of farnesoid $X$ receptor $b$ as a novel mammalian nuclear receptor sensing lanosterol. Mol Cell Biol 2003; 23: 864-72.

13 Huber RM, Murphy K, Miao B, Link JR, Cunningham MR, Rupar MJ, et al. Generation of multiple farnesoid-X-receptor isoforms through the use of alternative promoters. Gene 2002; 290: 35-43.

14 Zhang Y, Kast-Woelbern HR, Edwards PA. Natural structural variants of the nuclear receptor farnesoid $X$ receptor affect transcriptional activation. J Biol Chem 2003; 278: 104-10.

15 Pellicciari R, Costantino G, Fiorucci S. Farnesoid X Receptor: from structure to potential clinical applications. J Med Chem 2005; 48: 5383-403.

16 Laffitte BA, Kast HR, Nguyen CM, Zavacki AM, Moore DD, Edwards PA. Identification of the DNA binding specificity and potential target genes for the farnesoid X-activated receptor. J Biol Chem 2000; 275 : 10638-47.

17 Angelin B, Hershon KS, Brunzell JD. Bile acid metabolism in hereditary forms of hypertriglyceridemia: evidence for an increased synthesis rate in monogenic familial hypertriglyceridemia. Proc Natl 
Acad Sci U S A 1987; 84: 5434-8.

18 Bateson MC, Maclean D, Evans JR, Bouchier IA. Chenodeoxycholic acid therapy for hypertriglyceridaemia in men. $\mathrm{Br} J$ Clin Pharmacol 1987; 5: 249-54.

19 Bell GD, Lewis B, Petrie A, Dowling RH. Serum lipids in cholelithiasis: effect of chenodeoxycholic acid therapy. Br Med J 1973; 3: 520-3.

20 Beil U, Crouse JR, Einarsson K, Grundy SM. Effects of interruption of the enterohepatic circulation of bile acids on the transport of very low density-lipoprotein triglycerides. Metabolism 1982; 31: 438-44.

21 Crouse JR 3rd. Hypertriglyceridemia: a contraindication to the use of bile acid binding resins. Am J Med 1987; 83: 243-8.

22 Pullinger CR, Eng C, Salen G, Shefer S, Batta AK, Erickson SK, et al. Human cholesterol 7alpha-hydroxylase (CYP7A1) deficiency has a hypercholesterolemic phenotype. J Clin Invest 2002; 110: 109-17.

23 Sinal CJ, Tohkin M, Miyata M, Ward JM, Lambert G, Gonzalez FJ. Targeted disruption of the nuclear receptor FXR/BAR impairs bile acid and lipid homeostasis. Cell 2000; 102: 731-44.

24 Watanabe M, Houten SM, Wang L, Moschetta A, Mangelsdorf DJ, Heyman RA, et al. Bile acids lower triglyceride levels via a pathway involving FXR, SHP, and SREBP-1c. J Clin Invest 2004; 113: 140818.

25 Lu Y, Ma Z, Zhang Z, Xiong X, Wang X, Zhang H, et al. Yin Yang 1 promotes hepatic steatosis through repression of farnesoid $X$ receptor in obese mice. Gut 2014; 63: 170-8.

26 Zhang Y, Lee FY, Barrera G, Lee H, Vales C, Gonzalez FJ, et al. Activation of the nuclear receptor FXR improves hyperglycemia and hyperlipidemia in diabetic mice. Proc Natl Acad Sci U S A 2006; 103: 1006-11.

27 Calkin AC, Tontonoz P. Transcriptional integration of metabolism by the nuclear sterol-activated receptors LXR and FXR. Nat Rev Mol Cell Biol 2012; 13: 213-24.

28 Boulias K, Katrakili N, Bamberg K, Underhill P, Greenfield A, Talianidis I. Regulation of hepatic metabolic pathways by the orphan nuclear receptor SHP. EMBO J 2005; 24: 2624-33.

29 Huang J, Iqbal J, Saha PK, Liu J, Chan L, Hussain MM, et al. Molecular characterization of the role of orphan receptor small heterodimer partner in development of fatty liver. Hepatology 2007; 46: 147-57.

30 Parks DJ, Blanchard SG, Bledsoe RK, Chandra G, Consler TG, Kliewer $\mathrm{SA}$, et al. Bile acids: natural ligands for an orphan nuclear receptor. Science 1999; 284: 1365-8.

31 Xu J, Li Y, Chen WD, Xu Y, Yin L, Ge X, et al. Hepatic carboxylesterase 1 is essential for both normal and farnesoid $X$ receptor-controlled lipid homeostasis. Hepatology 2014; 59: 1761-71.

32 Pineda TI, Claudel T, Duval C, Kosykh V, Fruchart JC, Staels B. Bile acids induce the expression of the human perproliferator-activated receptor alpha gene via activation farnesoid $X$ receptor. Mol Endocrinol 2003; 17: 259-72.

33 Savkur RS, Bramlett KS, Michael LF, Burris TP. Regulation of pyruvate dehydrogenase kinase expression by the farnesoid $X$ receptor. Biochem Biophys Res Commun 2005; 329: 391-6.

34 Moore DD. Nuclear receptors reverse McGarry's vicious cycle to insulin resistance. Cell Metab 2012; 15: 615-22.

35 Hirokane H, Nakahara M, Tachibana S, Shimizu M, Sato R. Bile acid reduces the secretion of very low density lipoprotein by repressing microsomal triglyceride transfer protein gene expression mediated by hepatocyte nuclear factor-4. J Biol Chem 2004; 279: 45685-92.

36 Pan X, Zhang Y, Wang L, Hussain MM. Diurnal regulation of MTP and plasma triglyceride by CLOCK is mediated by SHP. Cell Metab 2010; 12: $174-86$.

37 Kast HR, Nguyen CM, Sinal CJ, Jones SA, Laffitte BA, Reue K, et al. Farnesoid X-activated receptor induces apolipoprotein $C$-II trans- cription: a molecular mechanism linking plasma triglyceride levels to bile acids. Mol Endocrinol 2001; 15: 1720-8.

38 Xiong $X$, Wang $X$, Lu Y, Wang E, Zhang Z, Yang J, et al. Hepatic steatosis exacerbated by endoplasmic reticulum stress-mediated downregulation of FXR in aging mice. J Hepatology 2014; 60: 84754.

39 Wu W, Zhu B, Peng X, Zhou M, Jia D, Gu J. Activation of farnesoid X receptor attenuates hepatic injury in a murine model of alcoholic liver disease. Biochem Biophys Res Commun 2014; 443: 68-73.

40 Lívero FA, Stolf AM, Dreifuss AA, Bastos-Pereira AL, Chicorski R, de Oliveira LG, et al. The FXR agonist 6ECDCA reduces hepatic steatosis and oxidative stress induced by ethanol and low-protein diet in mice. Chem Biol Interact 2014; 217: 19-27.

41 Liu X, Xue R, Ji L, Zhang X, Wu J, Gu J, et al. Activation of farnesoid $X$ receptor $(F X R)$ protects against fructose-induced liver steatosis via inflammatory inhibition and ADRP reduction. Biochem Biophys Res Commun 2014; 450: 117-23.

42 De Fabiani E, Mitro N, Gilardi F, Caruso D, Galli G, Crestani M. Coordinated control of cholesterol catabolism to bile acids and of gluconeogenesis via a novel mechanism of transcription regulation linked to the fasted-to-fed cycle. J Biol Chem 2003; 278: 39124-32.

43 Zieve FJ, Kalin MF, Schwartz SL, Jones MR, Bailey WL. Results of the glucose-lowering effect of WelChol study (GLOWS): a randomized, double-blind, placebo-controlled pilot study evaluating the effect of colesevelam hydrochloride on glycemic control in subjects with type 2 diabetes. Clin Ther 2007; 29: 74-83.

44 Stayrook KR, Bramlett KS, Savkur RS, Ficorilli J, Cook T, Christe ME, et al. Regulation of carbohydrate metabolism by the farnesoid $X$ receptor. Endocrinology 2005; 146: 984-91.

45 Cariou B, Duran-Sandoval D, Kuipers F, Staels B. Farnesoid X receptor: a new player in glucose metabolism? Endocrinology 2005; 146: 981-3.

46 Ma K, Saha PK, Chan L, Moore DD. Farnesoid X receptor is essential for normal glucose homeostasis. J Clin Invest 2006; 116: 1102-9.

47 Caron S, Huaman Samanez C, Dehondt H, Ploton M, Briand O, Lien $\mathrm{F}$, et al. Farnesoid $\mathrm{X}$ receptor inhibits the transcriptional activity of carbohydrate response element binding protein in human hepatocytes. Mol Cell Biol 2013; 33: 2202-11.

48 Cariou B, van Harmelen K, Duran-Sandoval D, van Dijk T, Grefhorst A, Bouchaert E, et al. Transient impairment of the adaptive response to fasting in FXR-deficient mice. FEBS Lett 2005; 579: 4076-80.

49 Cariou B, van Harmelen K, Duran-Sandoval D, van Dijk TH, Grefhorst $\mathrm{A}$, Abdelkarim $\mathrm{M}$, et al. The farnesoid $\mathrm{X}$ receptor modulates adiposity and peripheral insulin sensitivity in mice. J Biol Chem 2006; 281: 11039-49.

50 Kurosu H, Choi M, Ogawa Y, Dickson AS, Goetz R, Eliseenkova AV, et al. Tissue-specific expression of betaKlotho and fibroblast growth factor (FGF) receptor isoforms determines metabolic activity of FGF19 and FGF21. J Biol Chem 2007; 282: 26687-95.

51 Cyphert HA, Ge X, Kohan AB, Salati LM, Zhang Y, Hillgartner FB. Activation of the farnesoid $X$ receptor induces hepatic expression and secretion of fibroblast growth factor 21. J Biol Chem 2012; 287: 25123-38.

52 Lin Z, Tian H, Lam KS, Lin S, Hoo RC, Konishi M, et al. Adiponectin mediates the metabolic effects of FGF21 on glucose homeostasis and insulin sensitivity in mice. Cell Metab 2013; 17: 779-89.

53 Ryan KK, Tremaroli V, Clemmensen C, Kovatcheva-Datchary P, Myronovych A, Karns R, et al. FXR is a moleculartarget for the effects of vertical sleeve gastrectomy. Nature 2014; 509: 183-8.

54 Maneschi E, Vignozzi L, Morelli A, Mello T, Filippi S, Cellai I, et al. FXR activation normalizes insulin sensitivity in visceral preadipocytes of a 
rabbit model of MetS. J Endocrinol 2013; 218: 215-31.

55 Rizzo G, Disante M, Mencarelli A, Renga B, Gioiello A, Pellicciari R, et al. The farnesoid $X$ receptor promotes adipocyte differentiation and regulates adipose cell function in vivo. Mol Pharmacol 2006; 70 : 1164-73.

56 Abdelkarim M, Caron S, Duhem C, Prawitt J, Dumont J, Lucas A, et al. The farnesoid $X$ receptor regulates adipocyte differentiation and function by promoting peroxisome proliferator-activated receptorgamma and interfering with the Wnt/beta-catenin pathways. J Biol Chem 2010; 285: 36759-67.

57 Fuchs M. Non-alcoholic Fatty liver disease: the bile acid-activated farnesoid X receptor as an emerging treatment target. J Lipids 2012; 2012: 934396.

58 Fiorucci S, Rizzo G, Donini A, Distrutti E, Santucci L. Targeting farnesoid $X$ receptor for liver and metabolic disorders. Trends Mol Med 2007; 13: 298-309.

59 Ma Y, Huang Y, Yan L, Gao M, Liu D. Synthetic FXR agonist GW4064 prevents diet-induced hepatic steatosis and insulin resistance. Pharm Res 2013; 30: 1447-57.

60 Flatt B, Martin R, Wang TL, Mahaney P, Murphy B, Gu XH, et al. Discovery of XL335 (WAY-362450), a highly potent, selective, and orally active agonist of the farnesoid $X$ receptor (FXR). J Med Chem 2009; 52: 904-7.

61 Evans MJ, Mahaney PE, Borges-Marcucci L, Lai K, Wang S, Krueger
$\mathrm{JA}$, et al. A synthetic farnesoid $\mathrm{X}$ receptor (FXR) agonist promotes cholesterol lowering in models of dyslipidemia. Am J Physiol Gastrointest Liver Physiol 2009; 296: G543-52.

62 Zhang S, Wang J, Liu Q, Harnish DC. Farnesoid X receptor agonist WAY-362450 attenuates liver inflammation and fibrosis in murine model of non-alcoholic steatohepatitis. J Hepatol 2009; 51: 380-8.

63 Vignozzi L, Morelli A, Filippi S, Comeglio P, Chavalmane AK, Marchetta $\mathrm{M}$, et al. Farnesoid $\mathrm{X}$ receptor activation improves erectile function in animal models of metabolic syndrome and diabetes. J Sex Med 2011; 8: 57-77.

64 Mencarelli A, Renga B, Distrutti E, Fiorucci S. Antiatherosclerotic effect of farnesoid X receptor. Am J Physio Heart Circ Physiol 2009; 296: H272-81.

65 Cipriani S, Mencarelli A, Palladino G, Fiorucci S. FXR activation reverses insulin resistance and lipid abnormalities and protects against liver steatosis in Zucker (fa/fa) obese rats. J Lipid Res 2010; 51: 771-84.

66 Wang YD, Chen WD, Wang M, Yu D, Forman BM, Huang W. Farnesoid $X$ receptor antagonizes nuclear factor kappaB in hepatic inflammatory response. Hepatology 2008; 48: 1632-43.

67 Fiorucci S, Antonelli E, Rizzo G, Renga B, Mencarelli A, Riccardi L, et al. The nuclear receptor SHP mediates inhibition of hepatic stellate cells by FXR and protects against liver fibrosis. Gastroenterology 2004; 127: 1497-512. 